\title{
Performance evaluation for outdoor wireless scenarios based on IEEE 802.11b/g in the presence of co-channel interference
}

\author{
Roman A. Lara-Cueva, Michael S. Simba-Herrera \\ Departamento de Eléctrica, Electrónica y Telecomunicaciones, Grupe de Investigación en Sistemas Inteligentes \\ (WiCOM-Energy) and Ad Hoc Networks Research Center (CIRAD), \\ Universidad de las Fuerzas Armadas ESPE, Ecuador
}

\begin{tabular}{l}
\hline \hline Article Info \\
\hline Article history: \\
Received May 31, 2019 \\
Revised Aug 20, 2019 \\
Accepted Oct 12, 2019 \\
\hline
\end{tabular}

\section{Keywords:}

D-ITG

MATLAB

Medium access control Quality of Service (QoS)

Voice over internet protocol $\mathrm{WiFi}$

\begin{abstract}
Voice over Internet Protocol (VoIP) has evolved over the years, being a real-time service. VoIP has been coupled to different technologies, one of them is $\mathrm{WiFi}$, which is one of the most used for wireless local area networks in domestic and commercial environments. In this paper, we evaluate the performance of wireless scenarios by considering VoIP traffic, based on WiFi technology in conformance with IEEE $802.11 \mathrm{~b} / \mathrm{g}$ in interfered outdoor scenarios, by considering an intrusive injection traffic technique, for codecs G711(1 sample), G711(2 samples), G723, $\mathrm{G} 729$ (2 samples), and $\mathrm{G} 729$ (3 samples), related to the main metrics associated to Quality of Service (QoS) parameters. Our results show the best performance was obtained with the codecs G723 and G729(3 samples), obtaining up to 30 simultaneous voice connections with optimal values of delay, jitter and packet loss according to the recommendations given for VoIP by ITU-T, while the worst performance was obtained with the codec G711(2 samples), obtaining only 5 simultaneous voice connections, reaching an efficiency loss of around $18 \%$ in a co-channel interference scenario.
\end{abstract}

This is an open access article under the CC BY-SAlicense.

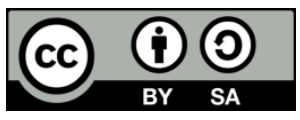

\section{Corresponding Author:}

Michael S. Simba-Herrera,

Departamento de Eléctrica, Electrónica y Telecomunicaciones,

Universidad de las Fuerzas Arma das ESPE,

Av. General Rumiñahui s/n Ambato, Sangolquí-Ecuador.

Email: mssimba@espe.edu.ec

\section{INTRODUCTION}

Technology has evolved in such way that messages, telephone calls, and video-conferences have become part of our daily lives [1]. In addition, the establishment of the Internet has allowed to Voice over Internet Protocol (VoIP) calls to grow remarkably, indeed according to Zion Research group, VoIP service has reached a total of 83 billion dollars in 2015, and it is estimated that by 2021 this service will reach a profit of 140 billion dollars, with a growth rate of 9.1\% [2]. VoIP is a viable alternative to the Public Switched Telephone Network (PSTN) due to its high utilization resources and low cost, achieving savings of up to $95 \%$ [3]. Initially, VoIP was used by wired connections, and now WiFi technology has allowed the use of VoIP over wireless connections, this evolution is known as Voice over WiFi (VoWiFi), which carries voice traffic in a basic area service, where the main disadvantage is related to some metrics associated to Quality of Service (QoS) as Throughput ( $\eta$ ), and Packet loss (PL), which could not guarantee real time transmissions in presence of interference. 
Previous research projects have covered the behaviour of WiFi technology [4-5], in conformance with the IEEE $802.11 \mathrm{~b} / \mathrm{g}$ standards by using analytical analysis [6], in which the paramete rs of the Medium Access Control (MAC) protocol are modified in order to obtain the voice capacity in an infrastructure network, and the maximum number of voice connections, reaching 19 voice connections with a satisfactory quality perceived by the user, meanwhile in [7] a simulation was performed to improve the quality of VoIP using signaling protocols, and codec schemes. Additionaly, in [8] was proposed an adaptive scheduling method for emissions based on network statistics, PL rate and packet availability. Otherwise, the performance evaluation of WiFi technology in an indoor environment was presented in [9-11], a handover analysis was performed in [12] with conventional Extended Service Set (ESS) infra structure with VoIP services and QoS assurance, and some methods to improve the quality of VoIP over a WLAN mesh were presented in [13], the capacity of switching mechanisms using failover mechanisms in a WAN and the quality of VoIP calls was studied in [14], also verifying the inherent limitations of the Distributed Coordination Function (DCF) to calculate the maximum number of VoIP clients a single Access Point (AP) can support, in which support 12 simultaneous VoIP calls considering G711 voice codec and 14 simultaneous VoIP calls considering G729 voice codec over a wireless network [15]. In [16] was showed low VoIP capacity in a WLAN and the unacceptable performance in the presence of coexisting traffic, another way to evaluate the behavior of the standard was by generating real calls by the software PBX-VoIP Asterisk, in [17] an outdoor environment was presented, and a total of simultaneous sessions were found that can be performed by using G729 voice codec reaching 25 simultaneous sessions, while with G711 voice codec 20 simultaneous sessions were obtained, also a traffic injection strategy was used in [18], by using Distributed Internet Traffic Generator (D-ITG), in where a laboratory environment was analyzed, in such environment was reached six clients connected wirelessly to an AP where the codec with the best stability was the G723.

To the best of our knowledge, no principled studies have performed the network capacity in a co-channel interfered outdoor scenarios, in such way the aim of this paper is to deployed an outdoor wireless network to evaluate the performance of the scenarios in the presence of co-channel interference. To accomplish this, we apply an intrusive injection traffic technique by considering the main metrics associated to QoS as $\eta$, delay ( $\delta$ ), Jitter, and PL, by considering VoIP tra ffic within three proposed scenarios. In the first scenario, three APs were implemented, each one separated by 120 degrees, creating three Basic Service Set (BSS) in the same frequency channel. The second scenario consisted of two APs placed in the same direction each one creating BSS in the same frequencies. The third scenario consisted of three APs positioned in the same direction, each of which creates a BSS on independent frequency channels.

\section{RESEARCH METHOD}

In this section we show theequipment and the applied methodology that was considered for the accomplishment of the experiment.Figure 1 shows the equipment deployed for the proposed scenarios, we used two and three AP working in the frequency band of $2.4 \mathrm{GHz}$ in conformance with IEEE $802.11 \mathrm{~b} / \mathrm{g}$ standards, these were connected via an Unshielded Twisted Pair (UTP) cable to a modem, that was responsible for distributing the traffic generated by the APs.The laptops used to perform VoIP traffic injections have installed Linux operating system (Ubuntu 10.04), one of them with an Core i5 processor that works at $2.60 \mathrm{GHz}$ with 6GB RAM memory was connected to one of the APs described above via WiFi, the other laptop with an Pentium CPU N3540 processor that works at $2.16 \mathrm{GHz}$ with 4GB RAM memory was connected to a different AP.

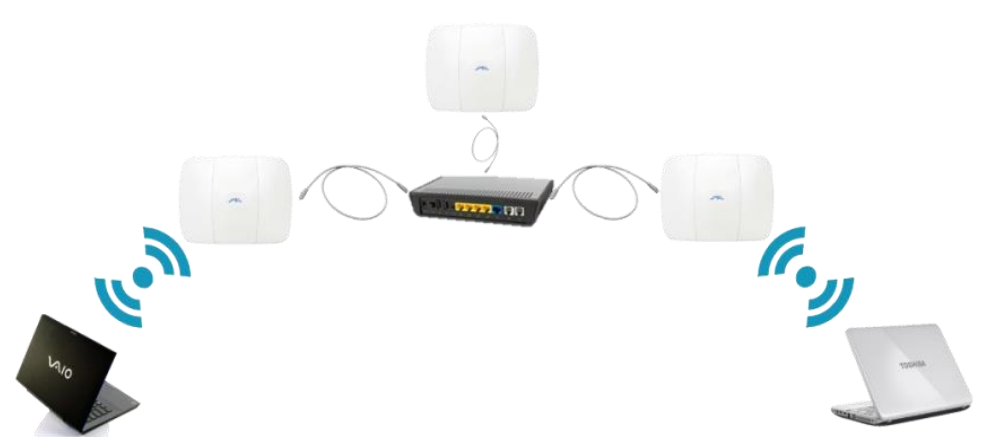

Figure 1. Equipment used for the different proposed scenarios 


\subsection{Performance with D-ITG}

In order to obtain the performance evaluation, we used an intrusive injection traffic technique by considering D-ITG software, which has 5 different predefined voice codecs: G711 of 1 and 2 samples, G723 and G729 of 2 and 3 samples as shown in Figure 2, also provides information of the main metrics associated to QoS, each flow represented a VoIP call, for this reason, the multi-flow sending option was used in the D-ITG software, which allows several flows to be carried out at the same time; for more inform ation about D-ITG please referred to [19-20]. In this way, several simultaneous multi-flow processes were carried out, starting with 5 multi-flow processes, then 10, 20, 30, 40, 50, until 60 multi-flow processes for each of the codecs. It should be noticed that in order to achieve a certain statistical validity in the results of traffic injections, 15 repetitions were performed for each multi-flow process as recommended in [21-22], so that the error in the direct measurements decreases at the time of the performance analysis.

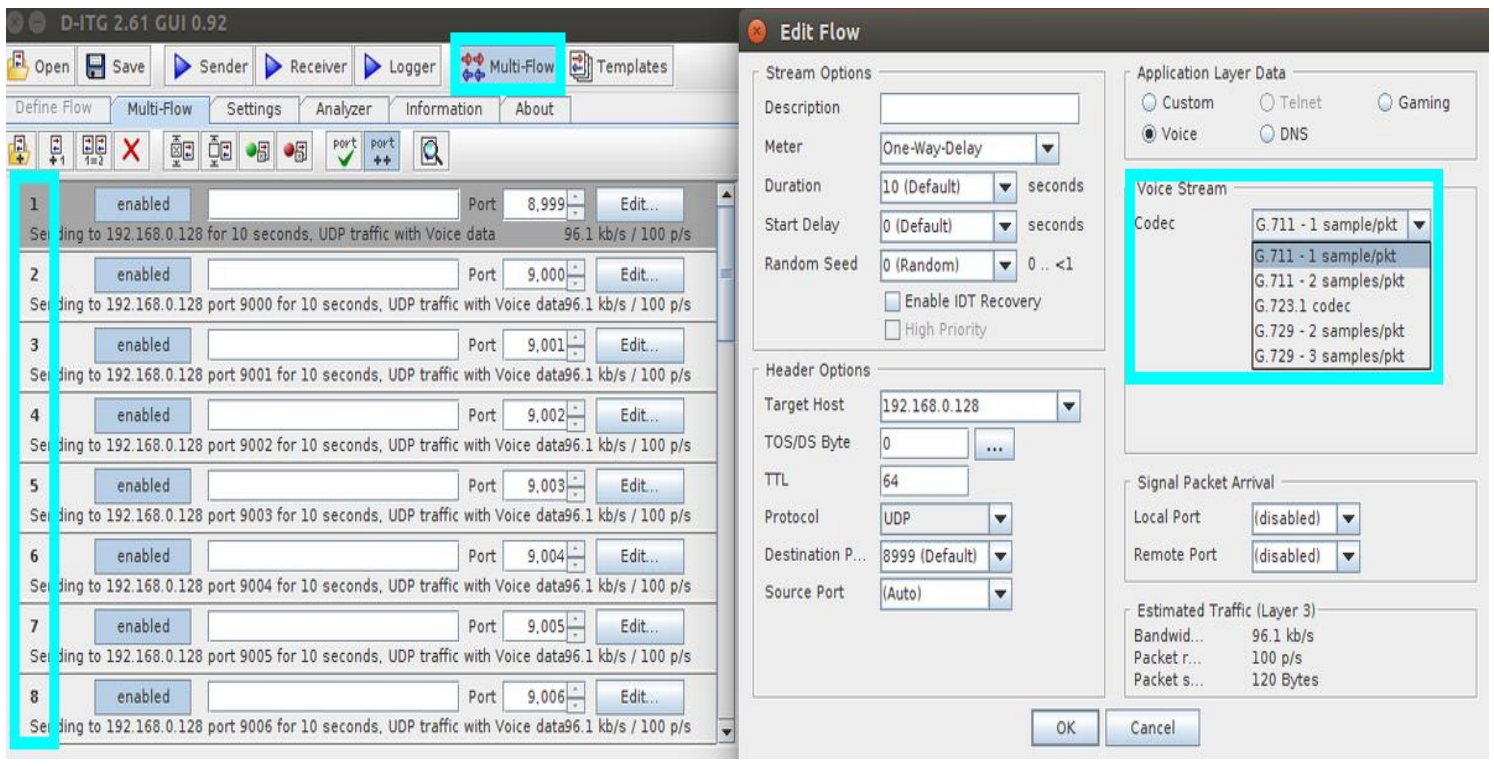

Figure 2. Voice codecs and multi-flows in D-ITG

\subsection{Scenarios}

In order to carry out the study, three different scenarios were considered, the network was deployed within Universidad de las Fuerzas Armadas-ESPEcampus in a telecommunications tower located on the roof of the Departamento de Eléctrica, Electrónica y Telecomunicaciones building, that consists of two floors; the APs were placed 5.5 meters from the base of the tower to perform traffic injections and to analyze their behavior by considering the main metrics associated to QoS parameters provided by the aforementioned software.

\subsubsection{Scenario 1}

The first scenario is shown in Figure 3, where three AP were implemented, each one separated by 120 degrees creating three independent BSS's, each one using the same frequency channel, for this scenario there existed only interference from other networks presented in the outdoor environment, since the beamwidth of the APs has only 18 degrees aperture, so that their radiation patterns do not overlap each other, in addition, the two laptops were connected to a different BSS to perform the traffic injections by using all the voice codecs of the software, first in one direction and then in the other, after all the traffic injections had been carried out, the data from the two directions were collected for analysis.

\subsubsection{Scenario 2}

The second scenario is showed in Figure 4, in the same way, two laptops were connected to a different AP, which are placed in the same direction, where each one created a different BSS that worked in the same frequency channel, for this scenario a co-channel interference was presented in addition to the interfering networks that may be presented when working in an outdoor environment. 


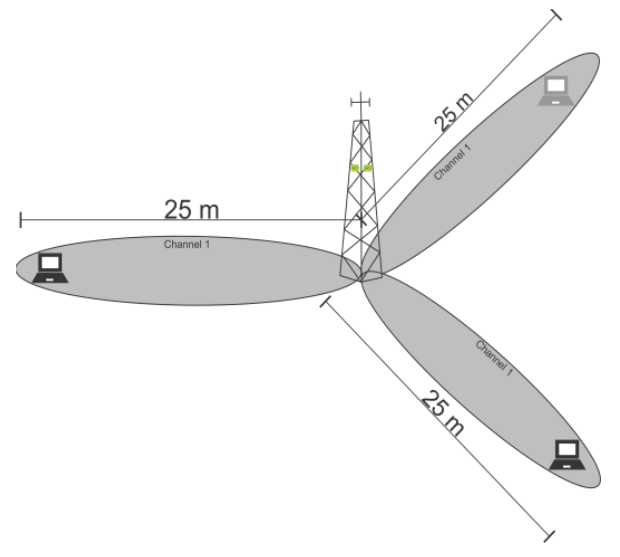

Figure 3. Scenario 1

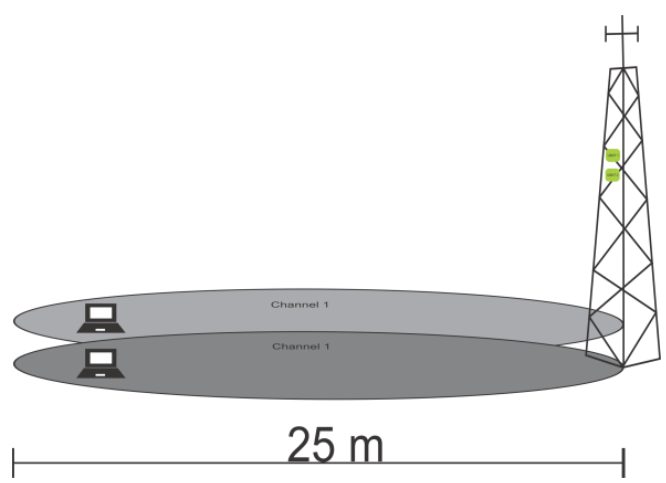

Figure 4. Scenario 2

\subsubsection{Scenario 3}

The third scenario is showed in Figure 5, for this scenario three APs were deployed which created three different BSS in independent frequency channels (ch. 1, ch. 6, and ch. 11), in addition, there were two laptops in the same way that in the previous scenarios, each one of them were connected to a different BSS, traffic injections were first performed by two BSS and then by the remaining BSS, the data from the three BSS were collected for analysis. For this scenario did not exist interference created by the APs since they were in independent channels, but there may be noise that interferes with traffic injections and also interference created by devices that may be present in an outdoor scenario.

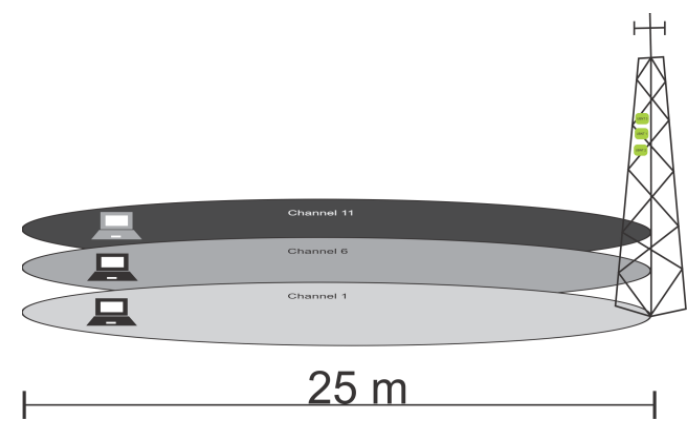

Figure 5. Scenario 3

The analysis of the traffic injections considering the main metrics associated to QoS was performed taking into account the data provided by D-ITG: $\delta, \eta$, Jitter and PL as shown in Figure 6, these data were classified according to the scenario and voice codec used, the analysis was performed in Matlab®. 


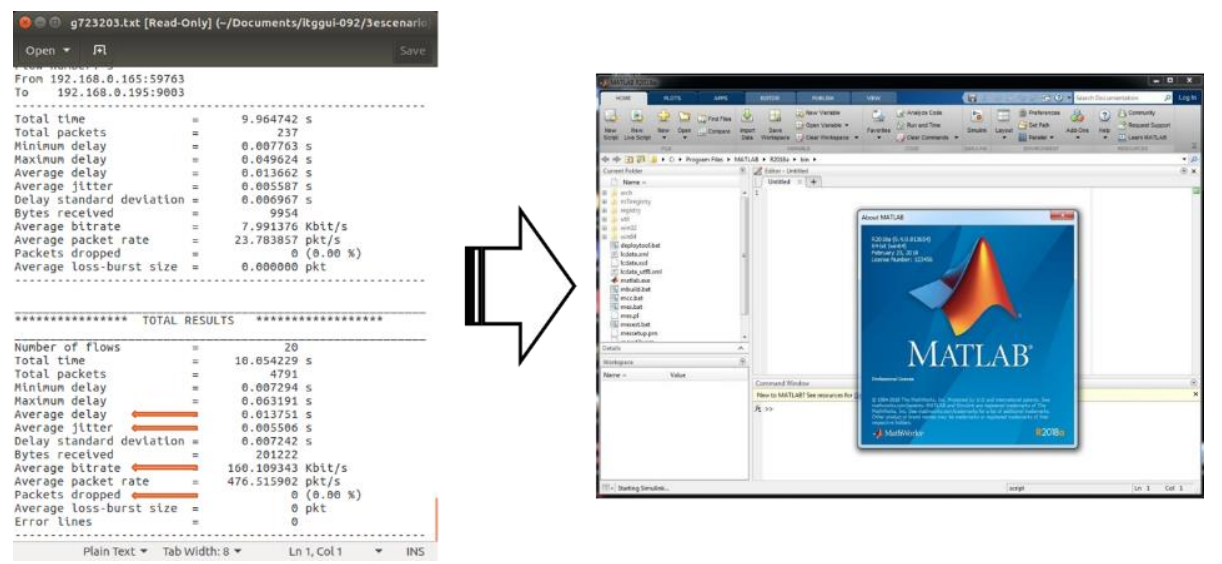

Figure 6. Data delivered by the software at the end of the multi-flows

\section{RESULTS AND DISCUSSION}

The analysis of the results was carried out according to the figures shown below, where the amount of simultaneous multi-flows made is called the number of voice connections, which are also shown in terms of percentage PL and $\eta$, while $\delta$ and Jitter are shown in milliseconds ( $\mathrm{ms}$ ). The three scenarios are described above, which were taken into account and the analysis was also made for each voice codec in order to know its behavior in each of the proposed scenario, bearing in mind the consideration of Qo $S$ parameters for VoIP were $\mathrm{PL}<1 \%, \delta<150 \mathrm{~ms}$ and Jitter $<30 \mathrm{~ms}$ [23-25].

For the first scenario, Figure 7 shows that with 5 voice connections all codecs satisfied QoS parameters, where the most efficient codec was G711(2 samples) with approximately $\eta$ of $80 \%$, when reaching the 10 voice connections the codecs G711(1 sample) and G723 satisfied the QoS parameters without any inconvenience, while the codecs G729(2 samples)and G729(3 samples) $\delta$ of $120 \mathrm{~ms}$ and $138 \mathrm{~ms}$ respectively, which approach the limit described in [23-25]; at 20 voice connections, only the codecs G723 and G729(3 samples) complied with the QoS parameters continuing with values close to the limit of $\delta$, this behavior was maintained until the 30 voice connections, meanwhile, from 40 to 60 voice connections, none of the codecs complied with the QoS parameters.
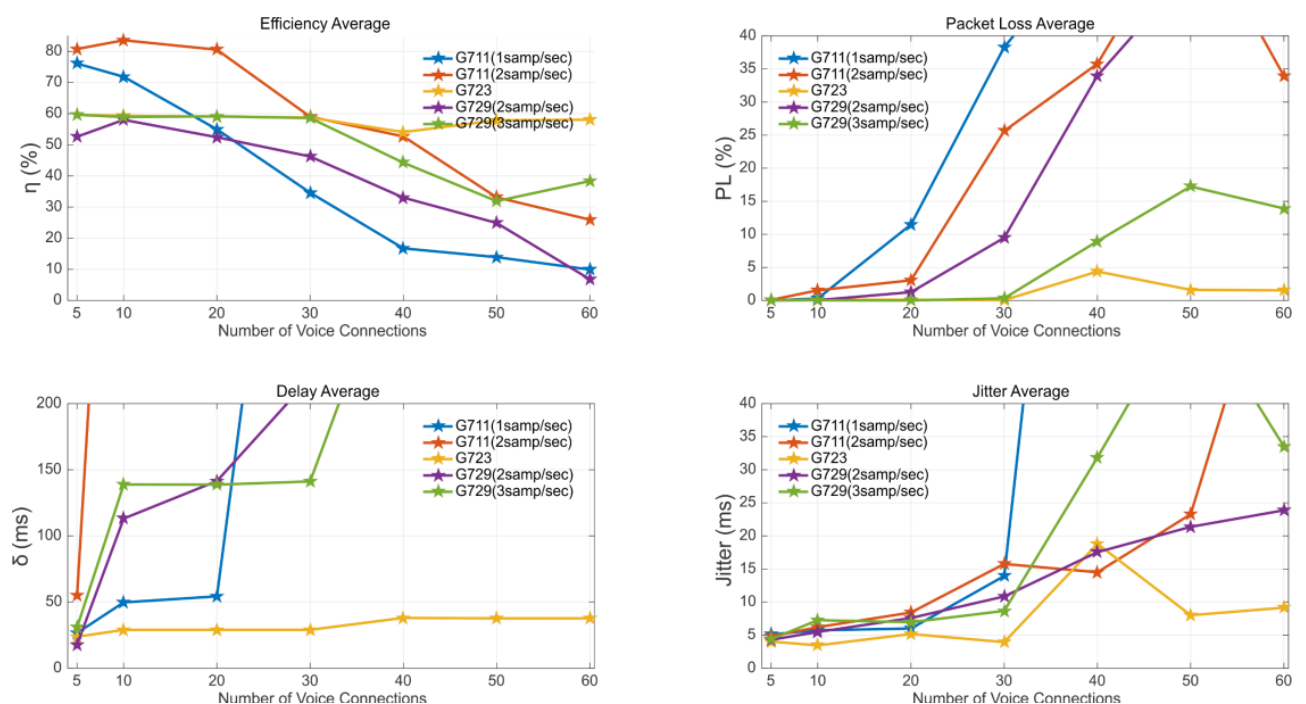

Figure 7. Results Scenario 1. (Blue line) Codec G711(1 sample), (Red line) Codec G711(2 samples), (Yellow line) Codec G723, (Purple line) Codec G729(2 samples), (Green line) Codec G729(3 samples)

The results of the second scenario are shown in Figure 8, in the same way as in the first scenario, with 5 voice connections, all codecs satisfied QoS parameters, where codec G711(2 sample) maintains the best efficiency with around $\eta$ of $80 \%$, by increasing voice connections to 10 , only codecs G723 
and G729(2 sample) satisfying QoS parameters, bearing in mind that codec G729(2 sample) had approximately $\delta$ of $130 \mathrm{~ms}$; reaching 20 voice connections, none of the codecs could satisfy all the parameters of QoS, the codec G729(3 samples) satisfied the parameters PL and Jitter but did not comply with the parameter $\delta$ as it had a value close to $200 \mathrm{~ms}$, while the G723 codec satisfied the $\delta$ and Jitter parameters but did not comply with the PL parameter which had an approximate value of $3 \%$, after 30 to 60 voice connections, all codecs had PL values above the ideal value, so none of the codecs comply with QoS parameters.
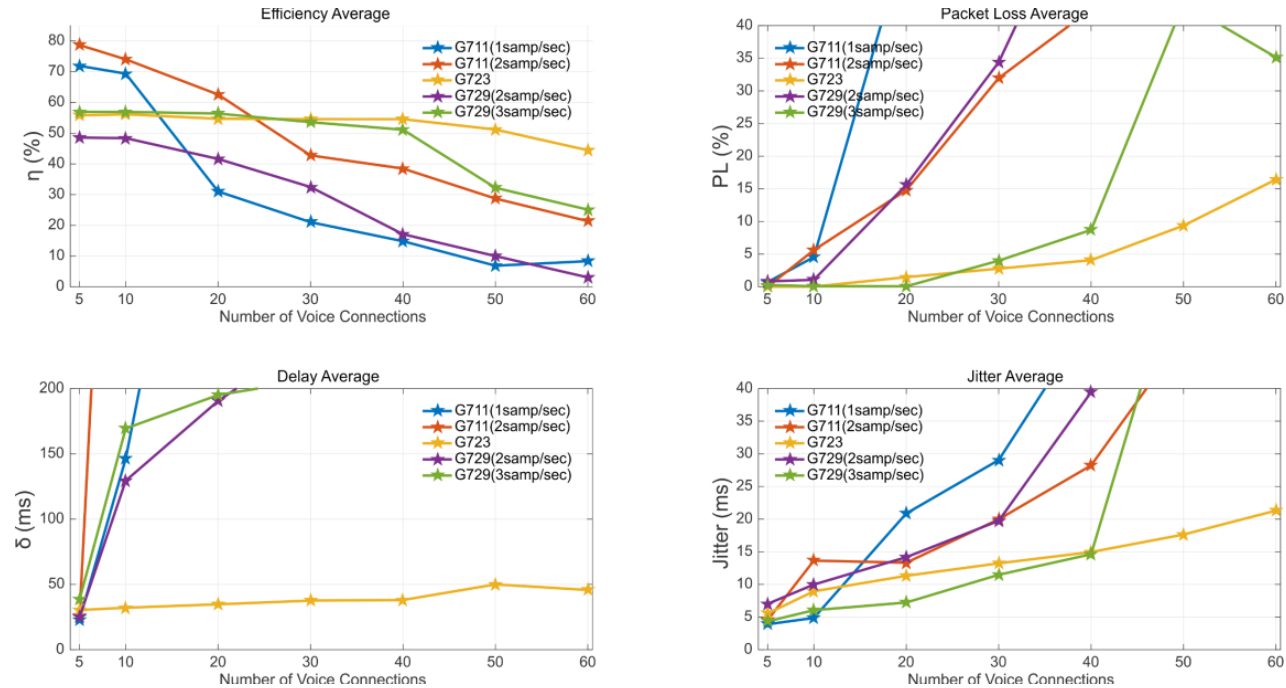

Figure 8. Results Scenario 2. (Blue line) Codec G711(1 sample), (Red line) Codec G711(2 samples), (Yellow line) Codec G723, (Purple line) Codec G729(2 samples), (Green line) Codec G729(3 samples)

Figure 9 shows the results of the third scenario, with 5 voice connections as in the previous scenarios, all codecs comply with QoS parameters and in the same way, the G711 codec ( 2 samples) remain s the most efficient, with around $\eta$ of $80 \%$, when the 10 voice connections were reach, three codecs continue to satisfied QoS parameters, these were the G711 codecs (1 sample), G723 and G729 (2 samples) where the latter had a value of $\delta$ close to the allowed range with a value of $145 \mathrm{~ms}$, while with 20 voice connections only the G723 codec satisfied the QoS parameters, from 30 to 60 voice connections, none of the codecs were able to satisfy all the QoS parameters, the G723 codec satisfied the parameters of $\delta$ and Jitter, but as in previous scenarios, it did not satisfy the required percentage of PL with values greater than $1 \%$.
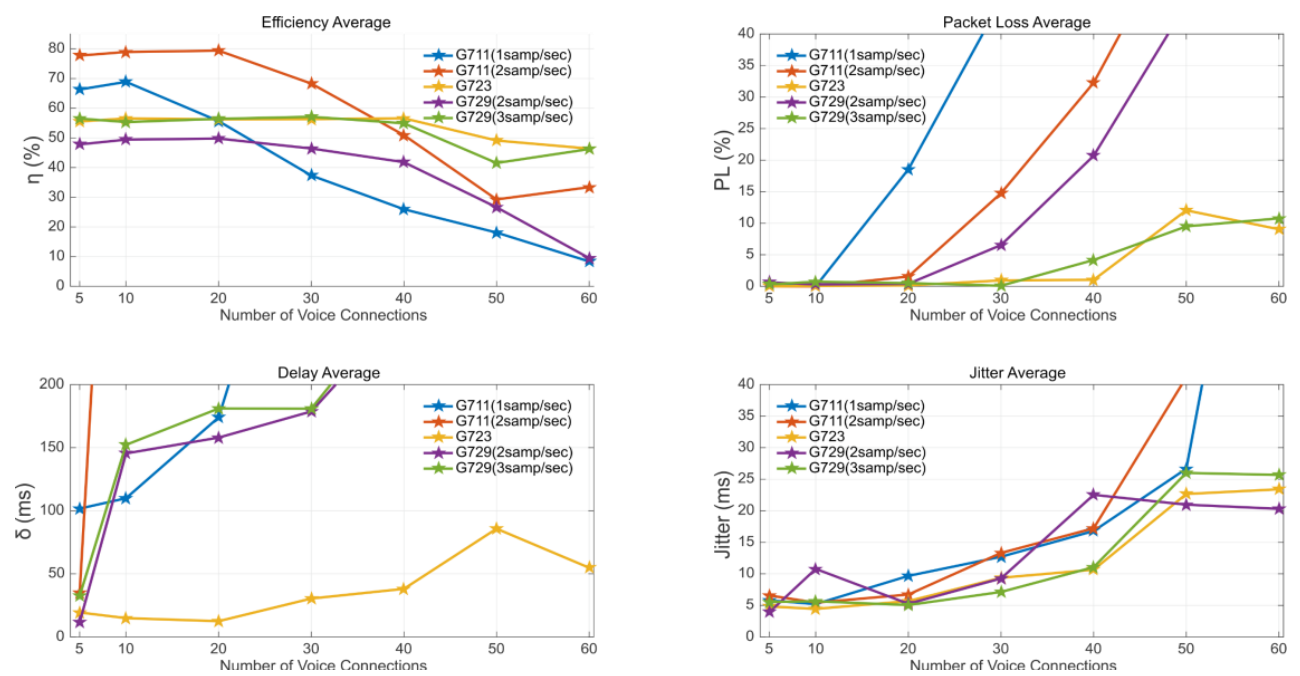

Figure 9. Results Scenario 3. (Blue line) Codec G711(1 sample), (Red line) Codec G711(2 samples), (Yellow line) Codec G723, (Purple line) Codec G729(2 samples), (Green line) Codec G729(3 samples) 
The behavior in the different scenarios of the codec G711(1 sample) may be appreciated in Figure 10, with values of $\delta$ of $25 \mathrm{~ms}$ for the 5 voice connections for the first and second scenarios, while the third scenario had a $\delta$ of $100 \mathrm{~ms}$, when reached the 10 voice connections the first scenario had a $\delta$ of $50 \mathrm{~ms}$ while the third scenario had a $\delta$ of $110 \mathrm{~ms}$ approximately; the best performance was reach in the first scenario, with lower values of $\delta$ compared to the third scenario, which were the scenarios that achieve 10 voice connections that complied with all QoS parameters.
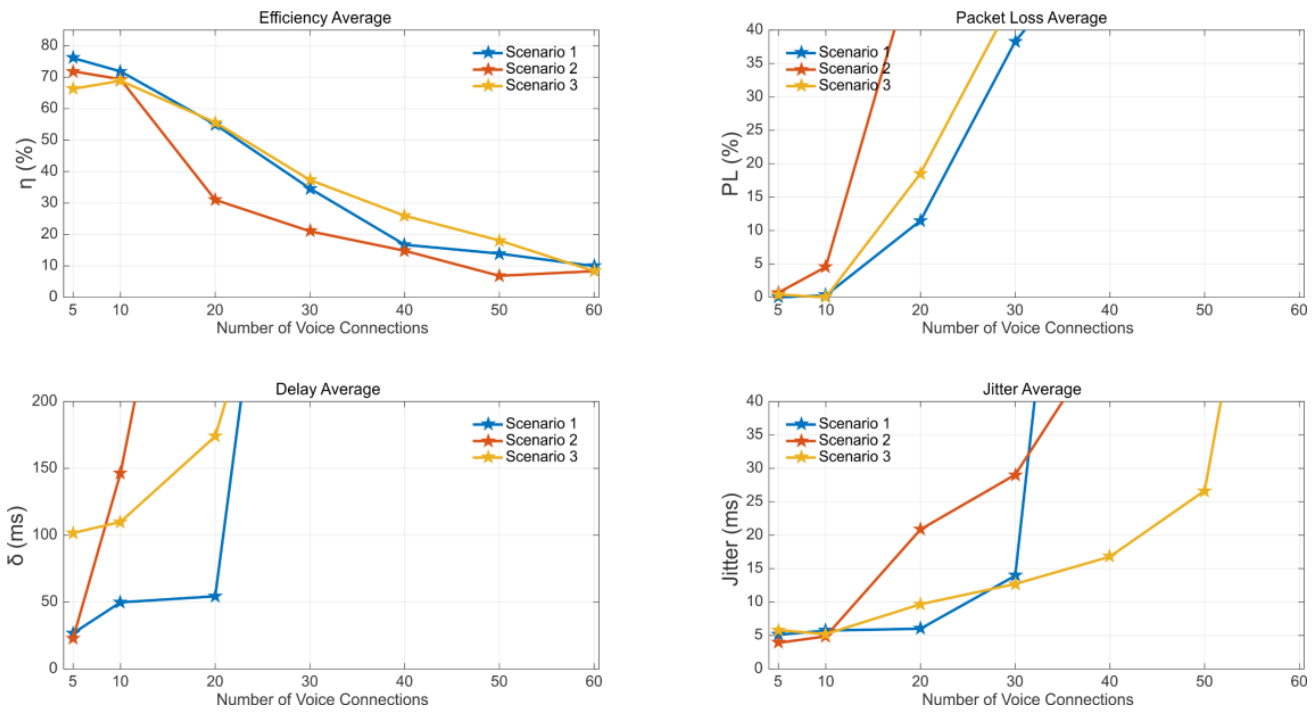

Figure 10. Results Codec G711(1 sample). (Blue line) Scenario 1, (Red line) Scenario 2, (Yellow line) Scenario 3

For the codec G711(2 samples) the behavior in the different scenarios is showed in Figure 11, this codec only managed to reach up to 5 simultaneous voice connections within the QoS parameters, in the three proposed scenarios it reached an efficiency of around $\eta$ of $80 \%$, where the best scenario was the second scenario with around $\delta$ of $25 \mathrm{~ms}$ followed closely by the third scenario with $\delta$ of $35 \mathrm{~ms}$, while the first scenario had $\delta$ of $55 \mathrm{~ms}$ approximately, as voice connections increase, the values of $\delta$ and PL increased rapidly, even surpassing $1000 \mathrm{~ms}$ and $50 \%$.
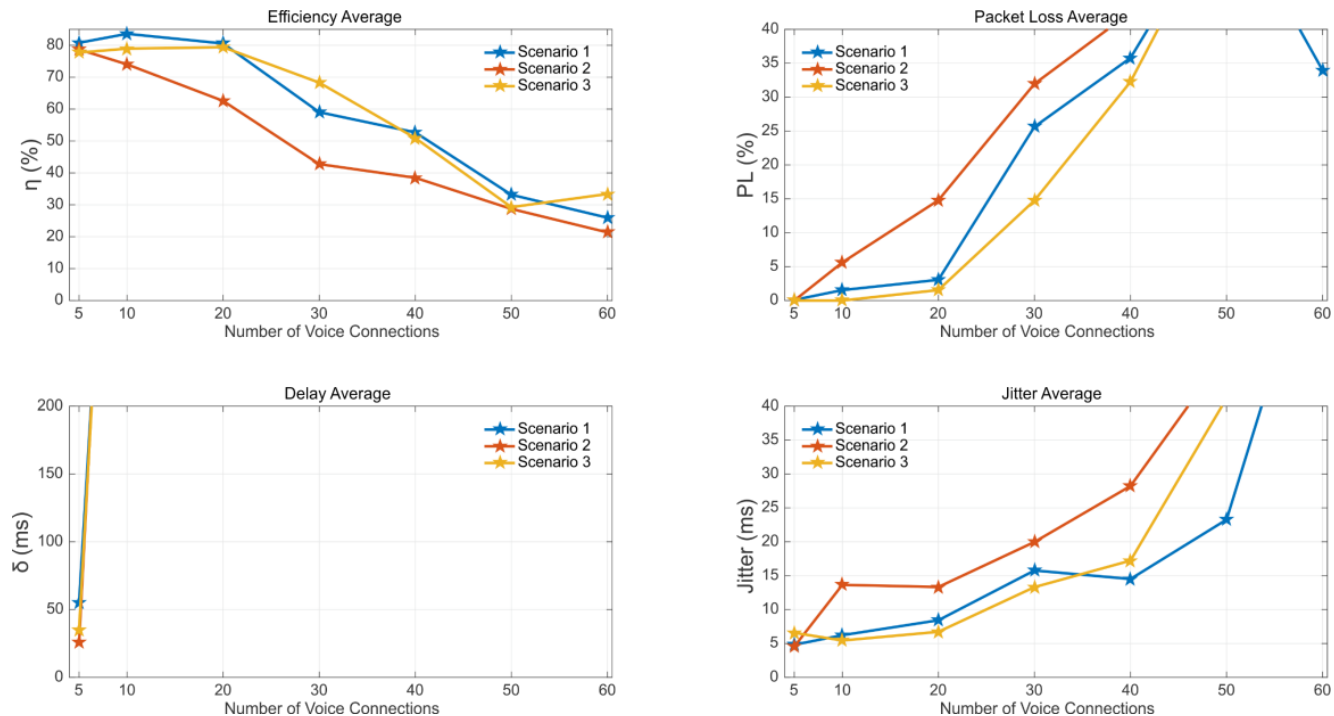

Figure 11. Results Codec G711(2 samples). (Blue line) Scenario 1, (Red line) Scenario 2, (Yellow line) Scenario 3 
The G723 codec was the only one in each of the scenarios proposed in terms of $\delta$ and Jitter was within the QoS parameters for all voice connections, the only problem within this codec lies in the percentages of PL, where the first scenario was the best performing reaching 30 voice connections with a value of less than PL of $1 \%$, in the third scenario reached 20 voice connections, while in the second scenario barely reached 10 voice connections, this is shown in Figure 12.
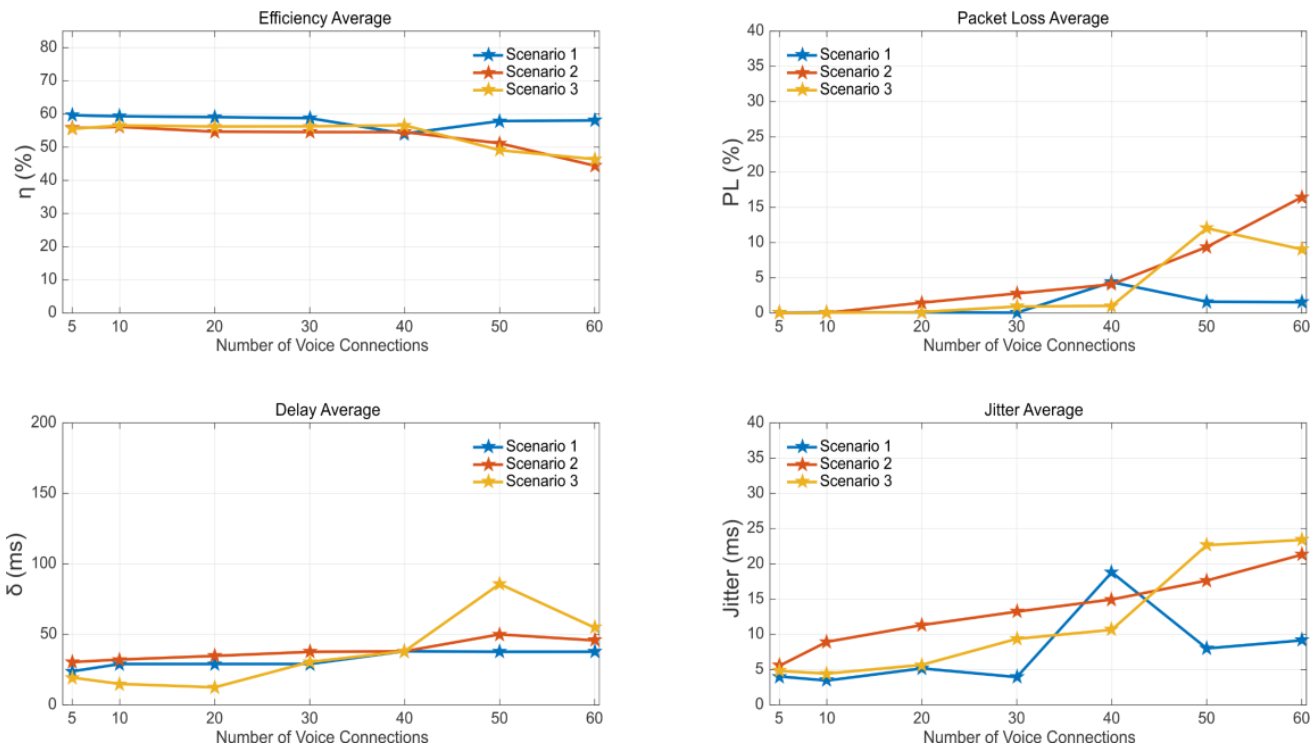

Figure 12. Results Codec G723. (Blue line) Scenario 1, (Red line) Scenario 2, (Yellow line) Scenario 3

Within the 3 proposed scenarios, codec G729(2 samples) had a similar behavior, reached only up to 10 voice connections, although with $\delta$ values quite close to the allowed limit, the lowest value of $\delta$ in 10 voice connections was in the first scenario with a value close to $110 \mathrm{~ms}$, while the highest value was in the third scenario with approximately $\delta$ of $145 \mathrm{~ms}$, Figure 13 shows the results of the different scenarios for this codec.
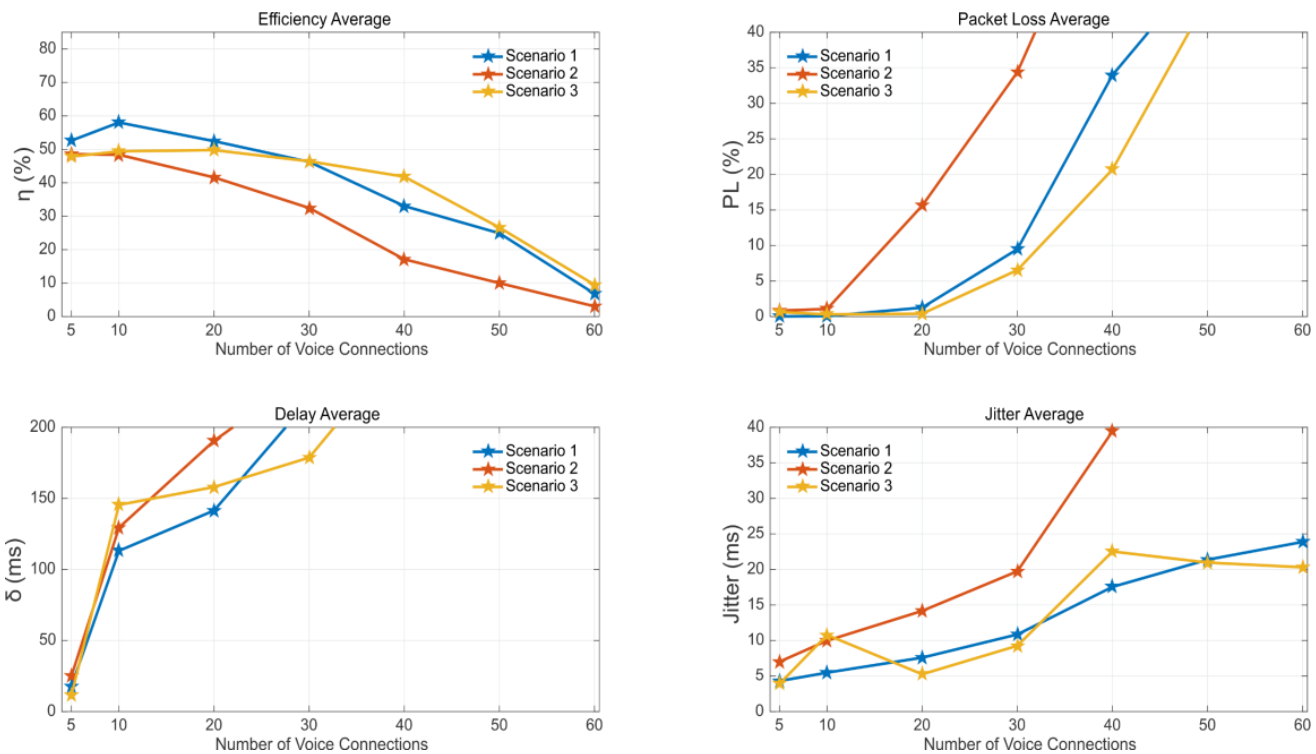

Figure 13. Results Codec G729(2 samples). (Blue line) Scenario 1, (Red line) Scenario 2, (Yellow line) Scenario 3 
Finally, for codec G729(3 samples) the scenario where the best results were obtained was the first scenario where up to 30 simultaneous voice connections are reached, similar to the previous codec the values of $\delta$ from 10 voice connections were high and maintain this constant until reaching 30 voice connections with a range between $120 \mathrm{~ms}$ and $141 \mathrm{~ms}$, this behaviormay be seen in Figure 14.
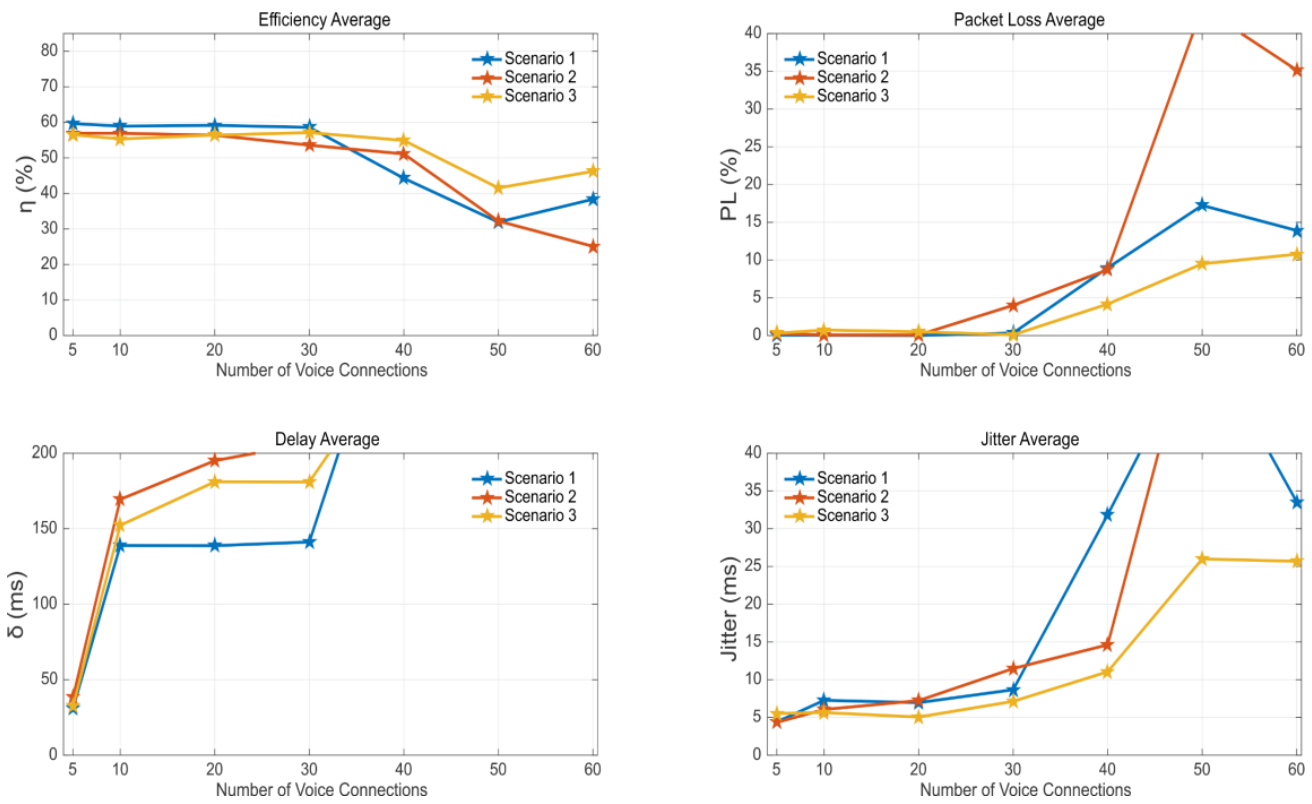

Figure 14. Results Codec G729(3 samples). (Blue line) Scenario 1, (Red line) Scenario 2, (Yellow line) Scenario 3

\section{CONCLUSION}

It was determined that the codec with the best performance considering the main metrics associa ted to QoS in the different test scenarios was the G723 codec, which achieved up to 30 simultaneous voice connections in the first scenario, 10 simultaneous voice connections for the second scenario and 20 simultaneous voice connections for the third scenario, on the other hand the codec with the worst behavior was the codec G711(2 samples), which achieved only 5 simultaneous voice connections in the three test scenarios, as well as in [17] where reached 5 voice connections in Central sub-scenario, which had 10 meters of distance between devices, working in the same frequency channel.

The scenario with the best performance considering the main metrics associated to QoS was the first scenario, where most of codecs managed to make 10 simultaneous voice connections without exceeding the parameters of QoS, it should be noted that in this scenario two codecs could reach up to 30 simultaneous voice connections, these are codecs G723 and G729(3 samples), higher than those reached in [17], where with the G729 codec reaching up to 25 voice connections in MED sub-scenario, which had 10 meters of distance between devices working in the same frequency channel; while the worst performing scenario was the second scenario with three codecs reaching only up to 5 simultaneous voice connections, these were codecs G711(1 sample), G711(2 samples), and G729(3 samples), a voice connection above for the G711 and G729 codecs in Enlace sub-scenario shown in [17], which had 128 meters of distance between two locations.

Taking into account the QoS parameters for VoIP that exist according to [23-25], it was possible to determine which are the limits for making simultaneous voice connections for each scenario, where the parameters that present abrupt changes between a certain number of voice connections and another were $\delta$ and PL. As these values increased, the Jitter also increased, although in a smaller proportion, while efficiency was also affected, being progressively less.Likewise, all interferences that are presented in an outdoor environment must be taken into account, since they cannot be avoided and affect the moment traffic intrusions are made in any of the scenarios, making the QoS values taken vary depending on the amount of interference that exists at that moment, where this can be appreciated most in the second scenario, when co-channel interference was presented in addition to interference found in an externalenvironment. 
Evaluating the different scenarios proposed, between the third and first scenario the efficiency was reduced by approximately $3.67 \%$, while comparing them with the second scenario wh ich presents co-channel interference, between the first and second scenario, the efficiency was reduced by approximately $18 \%$, and between the third and second scenario, the efficiency was reduced by approximately $18.3 \%$, noting that when using independent channels the efficiency was higher.Finally, as future work is proposed to evaluate the performance of VoIP outdoors with standards IEEE 802.11a, IEEE 802.11 n and IEEE 802.11 ac, work ing on the frequency of $5 \mathrm{GHz}$, so that the existing interference outdoors is less because it has a greater number of channels compared to the frequency of $2.4 \mathrm{GHz}$ with a high saturation in the channels, it would also be interesting to evaluate the performance of VoIP using WIMAX (Worldwide Interoperability for Microwave Access) technology

\section{ACKNOWLEDGEMENTS}

The authors gratefully acknowledge the contribution of Universidad de las Fuerzas Armadas ESPE for the economical support in the development of this project by Research Grants 2011 -PIT-WiLD and 2013-PIT-014.

\section{REFERENCES}

[1] W. Andocilla, M. Vallejo, "Integración de los servicios de voz sobre IP, aplicado a un caso de estudio," B.S. Thesis, Escuela Politécnica Nacional, Quito, Ecuador, March 2007.

[2] Market Research Store, "Global VoIP Services Market Set for Rapid Growth, to Reach Around USD 140 Billion By 2021," Market Research Store, May. 2, 2016. Accessed on: Feb. 26, 2019. [Online]. Available: https://www.marketresearchstore.com/news/global-voip-services-market-is-expected-to-reach-above-231

[3] Hipertextual, El papel de las centralitas virtuales en la internacionalización de empresas, Accessed on: Feb. 26, 2019. [Online]. Available: https://hipertextual.com/presentado-por/vozelia/centralitas-virtuales

[4] D. Villacis, F. Acosta, R. Lara, "Performance analysis of VoIP services over WiFi-based systems" Communications and Computing (COLCOM), Medellin, Colombia, pp. 1-6, May 2013.

[5] S. Suherman, "WiFi-friendly building to enable WiFi signal indoor,"Bulletin of Electrical Engineering and Informatics, vol. 7, no. 2, pp. 264-271, 2018.

[6] L. X. Cai, Xuemin Shen, J. W. Mark, L. Cai and Yang Xiao, "Voice capacity analysis of WLAN with unbalanced traffic," in IEEE Transactions on Vehicular Technology, vol. 55, no. 3, pp. 752-761, May 2006.

[7] O. Khalifa, R. Roslin, S. Bhuiyan, "Improved voice quality with the combination of transport layer \& audio codec for wireless devices,"Bulletin of Electrical Engineering and Informatics, vol. 7, no. 2, pp. 264-271, 2018.

[8] P. Chandran, C. Lingam, "A statistical approach to adaptive playout scheduling in voice over internet protocol communication,"International Journal of Electrical and Computer Engineering (IJECE), vol. 8, no. 5, pp. 2926-2933, 2018.

[9] R. Lara-Cueva, C. Morales and C. Fernández, "Performance evaluation of WiFi technology in conformance with IEEE 802.11b/n/ac and WDS for indoor environments," 2017 IEEE Colombian Conference on Communications and Computing (COLCOM), Cartagena, pp. 1-6, 2017.

[10] R. Lara-Cueva, D. Benítez, C. Fernández, and C. Morales, "Performance analysis of wireless network modes in conformance with ieee 802.11b and wds," in Asia-Pacific Conference on Computer Aided System Engineering, Quito, Ecuador, pp. 370-373, Jul 2015.

[11] M. A. Vallejo-Baldeón, D. N. Rodríguez and R. A. Lara-Cueva, "Performance evaluation of wireless network in conformance with IEEE 802.11n in co-channel interference scenarios," 2017 IEEE Colombian Conference on Communications and Computing (COLCOM), Cartagena, pp. 1-6, 2017.

[12] I. Prasetyo, M. Anif, A. Sriyanto, S. Pramono, S. Widodo, S. Hidayat, "Handover analysis of data and VoIP services in $802.11 \mathrm{~b} / \mathrm{g} / \mathrm{n}$ wireless LAN,"TELKOMNIKA (Telecommunication, Computing, Electronics and Control), vol. 12, no. 11, pp. 7832-7844, 2014.

[13] D. Niculescu, S. Ganguly, K. Kim and R. Izmailov, "Performance of VoIP in a 802.11 wireless mesh network," Proceedings IEEE INFOCOM 2006. 25TH IEEE International Conference on Computer Communications, Barcelona, pp. 1-11, 2006.

[14] H. Septama, A. Ulvan, R. Bestak, J. Hlavacek, "High available VoIP server failover mechanism in wide area network", TELKOMNIKA (Telecommunication, Computing, Electronics and Control), vol. 13, no. 2, pp. 739-744, 2015

[15] S. Garg and M. Kappes, "Can I add a VoIP call?," IEEE International Conference on Communications, 2003. ICC '03., Anchorage, AK, vo. 2, pp. 779-783, 2003.

[16] Wei Wang, Soung Chang Liew and V. O. K. Li, "Solutions to performance problems in VoIP over a 802.11 wireless LAN," in IEEE Transactions on Vehicular Technology, vol. 54, no. 1, pp. 366-384, Jan. 2005.

[17] M.Nuñez, C. Játiva, R. Lara, "Performance evaluation of VoIP technology in an extended service set, in concordance with IEEE 802.11g,"Sistemas y Telemática, vol. 15, no. 42, pp. 85-100, Oct. 2017.

[18] G. Agredo, J. Gaviria, "Experimental evaluation of the capacity of IEEE 802.11b to support VoIP services," Sistemas y Telemática, vol. 4, no.8, pp. 125-151, Oct. 2006. 
[19] A. Botta, A. Dainotti, A. Pescapè, "A tool for the generation of realistic network workload for emerging networking scenarios", Computer Networks (Elsevier), vol. 56, no. 15, pp 3531-3547, 2012.

[20] D-ITG, "Distributed Internet Traffic Generator," Accessed on: Feb. 18, 2019. [Online]. Available: http://www.grid.unina.it/software/ITG/manual/

[21] A. Posadas, "Determinación de Errores y Tratamiento de Datos," Accessed on: Feb. 27, 2019. [Online]. Available https://w3.ual.es/ aposadas/TeoriaErrores.pdf

[22] A. Kowalski, "Técnicas Auxiliares de Laboratorio," Accessed on: Feb. 27, 2019. [Online]. Available https://www.ugr.es/ andyk/Docencia/TEB/Errores.pdf

[23] T. Szigeti and C. Hattingh, "QoS Design Overview" in "End-to-End QoS network design: quality of service in LANs, WANs, and VPNs,"2nd ed. T. Szigeti, Ed. Indianapolis: Cisco Press, pp. 33-38, 2004.

[24] One-Way Transmission Time, document ITU-T Rec. G.114, International Telecommunication Union, Geneva, Switzerland, May 2003.

[25] P. Mehta and S. Udani, "Voice over IP," in IEEE Potentials, vol. 20, no. 4, pp. 36-40, Oct.-Nov. 2001.

\section{BIOGRAPHIES OF AUTHORS}
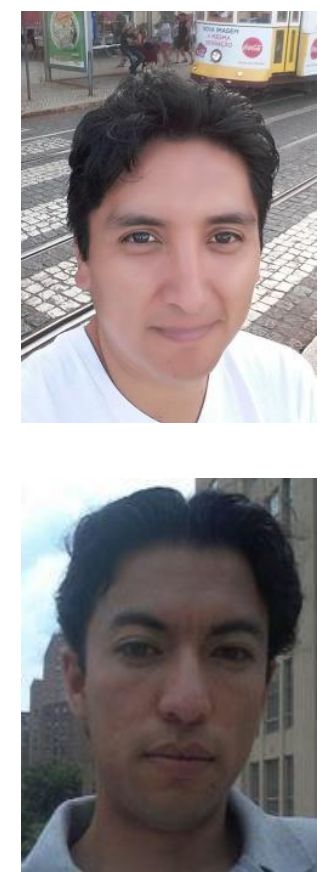

Román A. Lara-Cueva received the B.Eng. degree in electronic and telecommunications engineering from Escuela Politécnica Nacional, Quito, Ecuador, in 2001, the M.Sc. degree in wireless systems and related technologies from the Politecnico di Torino, Turin, Italy, in 2005, and the M.Sc. and Ph.D. degrees in telecommunication networks for developing countries from Rey Juan Carlos University, Fuenlabrada, Spain, in 2010 and 2015, respectively.In 2002, he joined the Departamento de Eléctrica, Electrónica y Telecomunicaciones, Universidad de las Fuerzas Armadas ESPE, Sangolquí, Ecuador, where he has been an Associate Professor since 2005 and a Full Professor since 2016. He has authored or coauthored 12 publicly funded research projects and directed seven of them. He currently heads the Ad Hoc Network Research Center and the Smart Systems Research Group (WiCOM-Energy). His research interests include digital signal processing, smart cities, wireless systems, machine learning theory, and volcano seismology.

Michael S. Simba-HerreraCandidate to Engineer in Electronics and Telecommunications at the Universidad de las Fuerzas Armadas - ESPE (Sangolquí, Ecuador). In 2018 he joined the Grupe de InvestigaciónenSistemasInteligentes (WiCOM-Energy) and Ad Hoc Networks Research Center (CIRAD) as research assistant. His main areas of interest in research are wireless systems and Voice over IP (VoIP). 